\title{
Modifying the yeast very long chain fatty acid biosynthetic machinery by the expression of plant 3- ketoacyl CoA synthase isozymes
}

Kenna Stenback

Harvard Medical School

Kayla Flyckt

Corteva Agriscience

Trang Hoang

University of Michigan

Alexis Campbell

Basil Nikolau ( $\nabla$ dimmas@iastate.edu )

lowa State University https://orcid.org/0000-0002-4672-7139

Article

Keywords:

Posted Date: December 14th, 2021

DOI: https://doi.org/10.21203/rs.3.rs-1153891/v1

License: (c) (i) This work is licensed under a Creative Commons Attribution 4.0 International License.

Read Full License 
1 Modifying the yeast very long chain fatty acid biosynthetic machinery by the expression of maize 3-ketoacyl CoA synthase isozymes

3 Kenna E. Stenback ${ }^{1, \# a}$, Kayla S. Flyckt ${ }^{1, \# b}$, Trang Hoang ${ }^{1, \# c}$, Alexis A. Campbell ${ }^{1, \# d}$, and Basil J.

Nikolau $^{1,2 *}$

${ }^{\# a}$ Current Address: Department of Biological Chemistry and Molecular Pharmacology, Harvard Medical School, Boston, Massachusetts, USA

${ }^{\mathrm{Ab} C}$ Current Address: Corteva Agriscience, Johnston, Iowa, USA

15

16

17

${ }^{\# c}$ Current Address: Department of Chemical Engineering, University of Michigan, Ann Arbor, Michigan, USA

\#dCurrent Address: School of Education, Iowa State University, Ames, Iowa, USA

18 *Corresponding Author 
Abstract

20 Eukaryotes express a multi-component fatty acid elongase to produce very long chain fatty acids

21 (VLCFAs), which are building blocks of diverse lipids. Elongation is achieved by cyclical

22 iteration of four reactions, the first of which generates a new carbon-carbon bond, elongating the

23 acyl-chain. This reaction is catalyzed by either ELONGATION DEFECTIVE LIKE (ELO) or 3-

24 ketoacyl-CoA synthase (KCS) enzymes. Whereas plants express both ELO and KCS enzymes,

25 other eukaryotes express only ELOs. We explored the KCS and ELO enzymatic redundancies

26 by expressing the former in yeast strains that lacked endogenous ELO isozymes. Expression of

27 the 26 maize KCS isozymes in wild-type, scelo2 or scelo3 single mutants did not affect VLCFA

28 profiles. However, five of these KCSs were capable of complementing the lethal scelo2; scelo3

29 double mutant. These rescued strains express novel VLCFA profiles reflecting the different

30 catalytic capabilities of the KCS isozymes. These novel strains offer a platform to explore the

31 relationship between VLCFA profiles and cellular physiology. 


\section{Introduction}

35 Very long chain fatty acids (VLCFAs) are defined as fatty acids with chain lengths that are 36 greater than 18 carbon atoms; in some organisms they can be up to 50 carbon atoms and longer

$37 \quad 1,2$. As building blocks for the assembly of more complex molecules, VLCFAs contribute to a 38 variety of cellular functions, including energy storage (e.g., triacylglycerols), biochemical

39 signaling (e.g., sphingolipids), and membrane structure (e.g., phosphoglycerolipids) ${ }^{3,4}$. In many

40 organisms, including plants, insects, and mammals, a subset of VLCFAs are also the building

41 blocks of an extracellular structure, the cuticle, which coats the exterior of these organism,

42 providing the primary physical barrier to the environment ${ }^{5,6}$. While VLCFAs account for a

43 minor portion of the fatty acid pool within cells, their importance is illustrated by the fact that the 44 absence of VLCFAs is lethal in fungi ${ }^{7}$, plants ${ }^{8,9}$ and animals ${ }^{8,9}$.

VLCFAs are generated by an ER-localized enzyme system, called fatty acid elongase (FAE) ${ }^{10,11}$

47 that utilizes preexisting fatty acids substrates, which are generated de novo from acetyl-CoA by a 48 fatty acid synthase (FAS) system ${ }^{12}$. Both the de novo FAS and the FAE systems utilize cyclical

49 iterations of Claisen condensation-reduction-dehydration-reduction reactions to elongate an acyl-

50 chain by 2-carbon atoms per cycle. In contrast to the FAS system, which utilizes acyl carrier

51 protein (ACP) bound acyl intermediates, the FAE system utilizes CoA-bound acyl intermediates

52 in these reactions.

54 Two types of non-homologous enzymes catalyze the initial Claisen condensation reaction of 55 FAE. One of these is the FAE1-like 3-ketoacyl-CoA synthases (KCS-type enzymes), and the 56 other is the ELONGATION DEFECTIVE-LIKE (ELO)-type enzymes. FAE1 was initially 57 identified in Arabidopsis ${ }^{13-15}$, and these KCS-type enzymes are only found in plants. ELO-type 
58 enzymes were initially identified in the yeast, Saccharomyces cerevisiae ${ }^{16,17}$, and they are more

59 widely distributed among eukaryotic organisms, including plants, fungi and animals ${ }^{17-19}$. The

60 reaction catalyzed by both of these enzymes involves the condensation between an acyl-CoA and

61 malonyl-CoA, generating a 3-ketoacyl-CoA product that is 2-carbon atoms longer than the initial

62 acyl-CoA substrate. The subsequent three reactions of each FAE cycle cumulatively chemically

63 reduces the 3-keto functional group to a methylene group, and the resulting acyl-CoA product is

64 the substrate for the next Claisen condensation reaction of the FAE cycle.

66 The occurrence of both KCS- and ELO-type enzymes in plants provides biochemical redundancy

67 in the generation of VLCFAs, which is even further enhanced by the genetic redundancy that

68 occurs in the genomes of these organisms. For example, the Arabidopsis and Zea mays (maize)

69 genomes appear to encode for 21 and $26 \mathrm{KCS}$ isozymes, and four and six ELO isozymes,

70 respectively ${ }^{18}$. This apparent redundancy of enzymes catalyzing the Claisen condensation

71 reaction of FAE is thought to be universal among terrestrial plant species, suggesting an

72 evolutionary advantage ${ }^{20,21}$.

74 In this study we explored the redundancy among the plant $K C S$-coding genes and the relationship

75 between KCS and ELO enzymes. We expressed each maize KCS isozyme in a wild-type yeast

76 strain, or in yeast strains that functionally lacked either individually or in combination two of the

77 three $E L O$-coding genes. Specifically, we evaluated the impact of expressing each of the 26

$78 \mathrm{ZmKCS}$ isozymes on the growth and fatty acid profiles of wild-type (WT), scelo2 and scelo3

79 single mutant strains, as well as the ability of these ZmKCS isozymes to genetically complement

80 the synthetically lethal scelo2; scelo3 double mutant strain. 
Methods

\section{$83 Z m K C S$ expression vectors}

84 Open reading frames (ORFs) of candidate $\mathrm{ZmKCS}$ sequences have been identified using

85 BLASTP ${ }^{22}$ by sequence homology with maize and Arabidopsis homologs ${ }^{18}$. These ORFs were

86 PCR amplified with appropriate primers from cDNA prepared from RNA isolated from the

87 maize inbred line, B73. RNA was purified from a number of different maize tissues using the

88 Qiagen RNeasy Kit (Hilden, Germany) (Supplementary Table 1), and RNA was converted to

89 cDNA using SuperScript ${ }^{\mathrm{TM}}$ Double-Stranded cDNA Synthesis Kit (Invitrogen, Carlsbad, CA).

90 Alternatively, if a $Z m K C S$ gene did not contain an intron, the $Z m K C S$ ORF was directly

91 amplified from B73 genomic DNA. These PCR reactions were conducted with Phusion high-

92 fidelity DNA polymerase (NEB, Ipswich, MA), and DNA products were isolated after agarose

93 gel electrophoresis using the Qiagen Gel Extraction Kit (Hilden, Germany). For three KCS

94 ORFs, (ZmKCS9, ZmKCS18 and ZmKCS19) sequences were codon optimized for expression in

95 yeast, and chemically synthesized as g-blocks (IDT, Coralville, IA).

97 A 'CACC' leader sequence required for entry cloning into $\mathrm{pENTR/dTOPO}$ vectors (Invitrogen;

98 Carlsbad, CA) was added to each KCS-coding fragment by PCR amplification or during DNA

99 synthesis. Assembled KCS-carrying pENTR/dTOPO plasmid-vectors were transformed into

100 One-Shot ${ }^{\mathrm{TM}}$ Mach1 ${ }^{\mathrm{TM}} \mathrm{T} 1$ competent $E$. coli cells using a standard protocol (Invitrogen,

101 Waltham, MA). LR clonase II (Invitrogen, Waltham, MA) was used to subclone KCS ORFs into

102 the appropriate Gateway yeast expression vectors, pAG423-GPD and pAG426-GPD ${ }^{23}$. All

103 plasmid-vectors were confirmed by sequencing (Iowa State University DNA Facility, Ames, IA) 
104 and E. coli strains harboring these plasmids were maintained on Luria-Bertani (LB) media

105 containing the appropriate antibiotics.

\section{Yeast strains and media}

108 Yeast cultures were grown using standard procedures ${ }^{24}$. Liquid precultures were grown to

109 stationary phase and then diluted into fresh media to an $\mathrm{OD}_{600}$ of 0.1 and subsequently grown at

$11030{ }^{\circ} \mathrm{C}$ with constant agitation at $250 \mathrm{rpm}$ for 24 hours. The parental strains BY4741 and BY4742

111 were maintained on YPD (Yeast Peptone Dextrose) media. Yeast strains carrying single gene-

112 deletion mutations were obtained from Open Biosystems (Hunstville, AL) and are listed in

113 Supplementary Table 2. These strains were maintained on YPD + $200 \mu \mathrm{g} / \mathrm{ml}$ Geneticin (G418;

114 Invitrogen, Waltham, MA). The double mutant yeast strain carrying the scelo2::KanMX $\mathrm{K}_{4}$ and

115 scelo3::HphMX6 disrupted alleles was maintained on YPD + $300 \mu \mathrm{g} / \mathrm{ml}$ Hygromycin (Goldbio,

116 St. Louis, MO). Yeast strains expressing maize $Z m K C S$ sequences were selected by their ability

117 to grow on synthetic defined minimal medium (SD) without the appropriate amino acid or

118 nucleobase. Strains that were genetically complemented with ZmKCS sequences were

119 maintained on SD-His media containing $1 \mathrm{mg} / \mathrm{ml}$ 5-fluoroorotic acid (5-FOA, Goldbio, St.

120 Louis, MO). Where appropriate, a strain that carried an empty expression vector used as a

121 control.

\section{Yeast genetics}

124 Plasmid transformation of yeast cells was by a standard lithium acetate protocol ${ }^{25}$. Each of the

$12526 \mathrm{ZmKCS}$ ORFs, cloned in the pAG426-GPD plasmid, were transformed into the BY4741 strain

126 that carried a scelo2 disrupted mutant allele, and into BY4742 strain that carried a scelo3

127 disrupted mutant allele (Supplementary Table 2). The ScELO3 gene was cloned with its native 
128 promoter sequence into a modified pAG416-GPD (low copy, URA3) plasmid backbone via In-

129 Fusion cloning (Takara Bio USA, Inc., Mountain View, CA). The modification of the pAG416

130 plasmid removed the GPD promoter from the vector backbone, so that the cloned ScELO3 gene

131 would be expressed by its native promoter. The resulting plasmid was transformed into a diploid

132 heterozygous strain, carrying scelo2::KanMX 4 and scelo3::KanMX 4 mutant alleles

133 (Supplementary Table 2). Sporulation of the resulting diploid strain was performed according to

134 Enyenihi and Saunders ${ }^{26}$, and the haploid scelo2::KanMX $\mathrm{K}_{4}$, scelo3::KanMX $\mathrm{K}_{4}$ double mutant,

135 which is normally lethal, was recoverable because it carried the ScELO3 expressing plasmid that

136 also carried the URA3 marker. This recovered double mutant was identified based on the

137 inability of the strain to grow on 5-FOA-containing media and was additionally confirmed by

138 PCR genotyping and DNA sequencing of the PCR product. The resistance cassette at the

139 scelo3::KanMX 4 locus was subsequently swapped with HphMX 6 (pAG32; Euroscarf, Frankfurt,

140 Germany), and this was confirmed by the ability of the strain to grow in the presence of 300

$141 \mu \mathrm{g} / \mathrm{ml}$ hygromycin, and by DNA sequencing of the scelo3::HphMX 4 allele. This strain was used

142 in plasmid shuffle experiments.

144 Complementation screen using plasmid shuffle

145 Plasmid shuffle experiments were performed with the scelo2::KanMX 4 , scelo3::HphMX 6 strain 146 that also carrier the pScELO3(URA3) plasmid using the method previously described by Truong

147 et al. ${ }^{27}$. In these experiments, the pScELO3(URA3) plasmid was shuffled with pAG423-GPD

148 expression vectors, each expressing one of the $26 \mathrm{ZmKCS}$ ORFs. In short, cells from a $600 \mu 1$

149 aliquot of a culture grown to an OD600 6 were pelleted and resuspended in water and spread

150 onto plates containing SD-His media containing $1 \mathrm{mg} / \mathrm{ml} \mathrm{5-FOA}$. Cultures were grown at $30^{\circ} \mathrm{C}$

151 for up to 21 days. To ensure that plates did not dry out during this time, they were placed in a 
152 sealed Tupperware container with damp paper towels, which maintained higher humidity. Plates

153 were examined weekly, and as colonies appeared, they were removed, and replica plated for

154 molecular characterizations. These characterizations included PCR confirmation of the loss of

155 the pScELO3 (URA3) plasmid, and PCR confirmation for the presence of the ZmKCS ORF. All

156 PCR products were sequenced for final confirmation.

\section{Strain growth analysis}

159 WT, and scelo2 or scelo3 mutant yeast strains each expressing individual ZmKCS isozymes were

160 grown in a BioTek microplate reader (Winooski, VT) for 36 hours at $30^{\circ} \mathrm{C}$ with constant

161 agitation, and growth was monitored by measuring OD600 every 30 minutes. Doubling time for

162 each culture was calculated using Gen5 data analysis software

163 (http://www.biotek.com/products/microplate_software/gen5_data_analysis_software.html).

164 Student's t-tests or Tukey's Honest Significant Difference tests were applied to statistically

165 evaluate differences in doubling time among strains.

166

167 The growth of the scelo2; scelo3 double mutant strains complemented by the expression of

$168 Z m K C S$ constructs were performed manually in liquid cultures, because these strains grew

169 slower than all other strains. Specifically, precultures were diluted to a starting OD600 0.2 in

$170250 \mathrm{ml}$ Erlenmeyer flasks containing $50 \mathrm{ml}$ of SD media supplemented with $1 \mathrm{mg} / \mathrm{ml}$ 5-FOA and

171 the necessary amino acids or nucleobases. Cultures were grown with constant agitation at 200

$172 \mathrm{rpm}$ at $30^{\circ} \mathrm{C}$. The OD600 of the cultures was monitored until cultures reached stationary phase of

173 growth. Growth of these complementing strains was also monitored on solid SD media

174 supplemented with $1 \mathrm{mg} / \mathrm{ml}$ 5-FOA and the necessary amino acids. In these experiments, a liquid

175 preculture of each strain was diluted to an OD600 0.3, and serially diluted 10-fold, and small 
176 triplicate aliquots of each dilution were spotted on the solid media. Plates were incubated at

177 either 23 or $30^{\circ} \mathrm{C}$ and imaged at 24-hour intervals.

179 Analysis of fatty acids

180 Fatty acids were extracted from an accurately weighed lyophilized cell pellet-aliquot, of between

1815 and $15 \mathrm{mg}$ each. Prior to extraction, the cell pellet was spiked with a known amount of

182 nonadecanoic acid internal standard (Sigma Aldrich, St. Louis, MO), and the mixture was

183 pulverized with glass beads (425-600 $\mu \mathrm{m}$; Sigma Aldrich, St. Louis, MO). Fatty acid methyl

184 esters (FAMEs) were produced by the addition of $1 \mathrm{~mL}$ of $5 \%$ sulfuric acid in methanol and

185 incubating at $85^{\circ} \mathrm{C}$ for $80 \mathrm{~min}$. After cooling, $2 \mathrm{~mL}$ of $0.9 \%$ (w/v) $\mathrm{NaCl}$ was added, and FAMEs

186 were recovered by extracting with two aliquots of 4:1 hexane:chloroform, which were pooled

187 and concentrated by evaporation. Samples were then derivatized with N,O-

188 Bis(trimethylsilyl)trifluoroacetamide with 1\% trimethylchlorosilane (BSTFA-TMSC, Sigma

189 Aldrich, St. Louis, MO) and analyzed with either GC-MS or GC-FID ${ }^{18}$.

\section{Transition Electron Microscopy (TEM)}

192 Cells for TEM imaging were collected from yeast cultures that were grown to mid-log phase. An

193 aliquot of such a culture was mixed with an equal volume of fixative $(2 \%(\mathrm{w} / \mathrm{v})$

194 paraformaldehyde and 6\% (w/v) glutaraldehyde in $0.2 \mathrm{M}$ sodium cacodylate buffer), and the

195 mixture was incubated at room temperature for 5 minutes. Fixed cells were collected by

196 centrifugation at $1,500 \mathrm{x} \mathrm{g}$ for 5 minutes, and the supernatant was removed. The cell pellet was

197 resuspended in 1\% (w/v) paraformaldehyde and 3\% (w/v) glutaraldehyde in $0.1 \mathrm{M}$ sodium

198 cacodylate buffer and incubated overnight at $4{ }^{\circ} \mathrm{C}$. Each subsequent step included re-suspension

199 and pelleting of cells at 1,500 x g for 5 minutes prior to solution changes. Samples were washed 
2 times with $0.1 \mathrm{M}$ cacodylate buffer for 10 minutes each, 2 times with distilled water for 10

201 minutes each, and then post-fixed with $1 \%(\mathrm{w} / \mathrm{v})$ aqueous potassium permanganate for 1 hour at

202 room temperature. Samples were then washed 3 times with deionized water for 15 minutes each,

203 and en bloc stained for 1 hour using 2\% (w/v) aqueous uranyl acetate. Samples were then

204 washed with distilled water for 10 minutes, and dehydrated through a graded ethanol series (25,

$20550,70,85,95,100 \%(\mathrm{v} / \mathrm{v}))$ for 1 hour for each step. Samples were further dehydrated with three

206 15-minute changes of acetone, and infiltrated with EmBed 812 formula (hard) for EPON epoxy

207 resin (Electron Microscopy Sciences, Hatfield, PA). This final infiltration involved 6-12 hour

208 incubations in graded resin:acetone mixtures $(1: 3,1: 1,3: 1)$, and final incubation in pure epoxy

209 resin. Cells suspended in the resin were placed into beem capsules and the resin was polymerized

210 at $70{ }^{\circ} \mathrm{C}$ for 48 hours. Fifty-nm thick microscopic sections were prepared using a Leica UC6

211 ultramicrotome (Leica Microsystems, Buffalo Grove, IL), and these were collected onto single

212 slot carbon film grids. Grids were post stained for 30 minutes with $2 \%(w / v)$ aqueous uranyl

213 acetate, followed with a 30-minute treatment with Reynold's lead stain to enhance contrast. TEM

214 images were collected using a 200kV JEOL JSM 2100 scanning transmission electron

215 microscope (Japan Electron Optics Laboratories, USA, Peabody, MA), equipped with a GATAN

216 One View 4K camera (Gatan inc., Pleasanton, CA). Cell wall thickness was measured using

217 ImageJ 1.53a (https://imagej.nih.gov/ij/).

\section{Statistical methods}

220 Data are presented as mean values with standard error. Comparisons with a p-value $<0.05$ were

221 considered statistically significant. Data processing was performed in Microsoft Excel (2021).

222 Statistical tests were carried out using JMP Pro 16 (SAS Institute Inc.; Cary, NC). Data were 
223 evaluated for statistical rigor based upon a 2-sided unpaired $t$ test or ANOVA followed by post-

224 hoc Tukey's Honest Significant Difference test (Supplementary Table 3). Principal component

225 analysis (PCA) was performed using JMP Pro 16.

\section{Results}

\section{ZmKCS expression in the WT BY4742 background}

228 Initial characterizations involved the individual expression of the $26 \mathrm{ZmKCS}$ sequences in a yeast

229 strain that contained an intact, WT FAE system. Expression of 11 of the ZmKCSs caused a

230 statistically significant abatement of growth as compared to the recipient strain. Notably, five of

231 these strains, expressing ZmKCS9, ZmKCS18, ZmKCS19, ZmKCS24, or ZmKCS27, showed a

$23225 \%$ increase in doubling times, and with the other six strains (ZmKCS2, ZmKCS3, ZmKCS10,

$233 Z m K C S 11, Z m K C S 21$, and ZmKCS23), doubling time was increased by 5-10\% (Supplementary

234 Fig. 1a).

235

236 Fatty acid analyses of the individual strains expressing each of the ZmKCS isozymes identified

237 fatty acids that normally accumulate in WT yeast strains ${ }^{28}$, ranging between 10 -carbon and 26-

238 carbon chain lengths, including monounsaturated fatty acids and fatty acids that are hydroxylated

239 at the 2-position (Supplementary Fig. 1c and e). These fatty acids were evaluated as products of

240 either de novo fatty acid biosynthesis, produced by the yeast FAS1/FAS2 complex that generates

241 fatty acids of up to 18-carbon chain length (Supplementary Fig. 1b and c), or as products of the

242 FAE system that generates the longer chain fatty acids of up to 26-carbon chain length

243 (Supplementary Fig. 1d and e) ${ }^{29}$. Among the 26 ZmKCS expressing strains, only the expression

244 of $Z m K C S 2$ and $Z m K C S 3$, showed statistically significant quantitative or qualitative changes in

245 the fatty acid profiles. Specifically, expression of ZmKCS2 increased the FAE-generated

246 products, including statistically significant increases in 24 and 26-carbon fatty acids, whereas the 
247 expression of $\mathrm{ZmKCS} 3$ induced a quantitative increase (by 25\%) of the products derived from

248 FAS, without qualitatively affecting the fatty acid profiles that were produced by the recipient

249 strain. The expression of all the other $\mathrm{ZmKCS}$ proteins had no significant quantitative effects,

250 and only had subtle effect on the FAE- or FAS-derived product profiles (Supplementary Table

251 S3).

252

253 ZmKCS expression in the yeast scelo2 mutant background

254 All 26 ZmKCS isozymes were individually expressed in a yeast mutant strain that lacked a

255 functional scelo2 gene. The scelo2 mutation did not affect the growth of the strain as compared

256 to the strain that carried a WT yeast FAE. Moreover, adding each ZmKCS into this scelo2

257 mutant strain also did not affect the growth of the strain. Indeed, except for the ZmKCS21-

258 expressing strain, there was no significant change in growth rates of these strains as compared to

259 the strain that carried the WT yeast FAE system (Supplementary Fig. 2a).

261 Fatty acid analyses of these strains show that the most significant impact of the scelo2 mutation

262 is the hypo-accumulation of FAE-generated VLCFAs (i.e., 50\% reduction as compared to the

263 WT strain) (Supplementary Fig. 2d). Most notably are the significant reductions in C20- and

264 C26-carbon fatty acids ( 85\% decrease as compared to WT). Despite these changes in VLCFA

265 accumulation, as expected the disruption of the scelo 2 gene had only minor statistically

266 significant effects on the fatty acids produced by the FAS system; the most significant

267 modulation being the increased accumulation of minor FAS constituents, including C15- and

268 C17-carbon fatty acids, as well as their 2-hydroxy derivatives (5-10 fold increase, Supplementary

269 Table 3). 
271 The individual expression of the 26 ZmKCS genes in this scelo2 mutant background did not

272 significantly change the VLCFA content as compared to the recipient strain and caused only

273 subtle, insignificant changes in the relative abundances of individual VLCFAs (Supplementary

274 Fig. 2e). Furthermore, the expression of individual ZmKCS genes in this mutant background had

275 minor or statistically insignificant changes in the FAS-derived products (Supplementary Fig. 2b

276 and c, Supplementary Table 3).

277

278

279

280

281

282

283

284

285

286

287

288

289

290 291 hydroxylated derivatives.

\section{ZmKCS expression in the yeast scelo3 mutant background}

The lack of a functional scelo3 gene did not significantly affect the growth of the yeast strain (Supplementary Fig. 3a). However, the expression of 11 specific ZmKCS genes in this mutant strain significantly reduced growth, increasing doubling time by $10-40 \%$. These strains were also analyzed to assess their ability to produce fatty acids. As previously established ${ }^{16}$, the scelo3 mutation caused a large ( 10-fold), quantitative increase in the accumulation of VLCFAs as compared to the WT control strain (Supplementary Fig. 3d). Most notably, this was affected by the loss of the 26-carbon VLCFA and its hydroxylated derivative, and this is coupled with a reciprocal increase in the accumulation of 20-carbon, and 22-carbon VLCFAs and 2-hydroxyderivatives (Supplementary Fig. 3e). With the exception of the ZmKCS15-expressing strain, the expression of the other ZmKCSs in this scelo3 mutant did not change this profile. On the other hand, the ZmKCS15-expressing strain was able to produce statistically significant increased quantity of 24-carbon VLCFAs, and detectable quantity of 26-carbon VLCFAs and their

293 Parallel evaluation of the FAS-generated fatty acids produced by these strains, indicates that the 294 majority of the ZmKCS-expressing strains (21 strains) did not change the accumulation of these 
295 fatty acid products. The other five ZmKCS-expressing strains had minor, but statistically

296 significant effects on the accumulation of these FAS-generated fatty acid products

297 (Supplementary Fig. 3b and c); ZmKCS2 and ZmKCS18 increased these fatty acids by 15\%,

298 and the expression of ZmKCS24, ZmKCS25, and ZmKCS27 lead to 30\% decrease in FAS

299 products. In the latter three strains, a significant reduction in 18:1 fatty acid was observed (50-

$30070 \%$ reduction as compared to the scelo3 mutant strain).

302 Genetic complementation of the synthetically lethal scelo2; scelo3 double mutant by $303 \quad Z m K C S$ expression

304 The ability of the 26 ZmKCS genes to complement the synthetic lethality of the scelo2; scelo3

305 double-mutant was determined by a plasmid-shuffle strategy. In these experiments we expressed

306 each ZmKCS isozyme in a strain that carried an episomal wild-type ScELO3 allele, which

307 maintained the viability of the otherwise synthetically lethal scelo2; scelo3 double-mutant strain.

308 Because the episomal wild-type ScELO3 allele also carried the URA3 selection marker, growing

309 these strains on 5-FOA-containing media counter-selected the episomal ScELO3 allele, and

310 strains that expressed a ZmKCS capable of complementing the scelo2; scelo3 double mutant

311 lethality were recovered. These experiments resulted in the recovery of five ZmKCS genes

312 (ZmKCS2, ZmKCS4, ZmKCS11, ZmKCS15, and ZmKCS20), demonstrating their ability to

313 complement the lethality associated with the scelo2; scelo3 double mutant (Fig. 1).

315 Even though the recovered strains were viable, they each suffered a significant growth penalty as

316 compared to the WT strain, increasing doubling time by between 2- and 4-fold as compared to

317 the BY4741 and BY4742 WT strains, and the scelo2; scelo3 + pScELO3 control strain (Fig. 2a). 
318 The ZmKCS2 complementing strain had the longest doubling time; four-fold increase as

319 compared to the BY4741 control. The ZmKCS15 complementing strain had the shortest doubling

320 time, although it was still 2- to 3-fold longer than the control strains (Table 3).

321

322 Additionally, the growth of these ZmKCS complementing strains were assessed on solid media,

323 grown at either $23{ }^{\circ} \mathrm{C}$ or $30^{\circ} \mathrm{C}$ (Supplementary Fig. 4). Similar to the growth observed in liquid

324 media, all the complementing strains grew slower than the BY4741 strain and of these, the

$325 \mathrm{ZmKCS} 15$-complemented strain was the fastest growing on solid media (Fig. 2B). At $23{ }^{\circ} \mathrm{C}$, the

326 growth of all the strains were initially delayed, with the greatest impact being on the ZmKCS4

327 complement, which showed only minimal growth even after a 168-h period (Fig. 2B).

328

329 Fig. 3 compares the cellular ultrastructure of the five ZmKCS complementing strains to those of

330 the BY4741, BY4742, and scelo2; scelo3 + pScELO3 control strains (Fig. 3a-c). The most

331 consistent difference between these strains is the increased occurrence of fragmented vacuoles in

332 all the ZmKCS complementing strains (Fig. 3d-h), and the alteration in the thickness of the cell

333 wall. The latter ultrastructural feature was quantifiable, and these data indicate that the thickness

334 of the cell wall is increased by $\sim 35 \%$ in the ZmKCS4-complemented strain, whereas the

$335 \mathrm{ZmKCS15-complemented} \mathrm{strain} \mathrm{has} \mathrm{a} \mathrm{cell} \mathrm{wall} \mathrm{that} \mathrm{is} \sim 30 \%$ thinner from the control strains

336 (Supplementary Fig. 5)

338 The fatty acid profiles of the five $Z m K C S$-expressing strains that complement the lethality of the

339 scelo2; scelo3 double mutant were determined and compared to each other and to the control

340 strains. As may be expected, there are only subtle quantitative or qualitative changes in the fatty 
341 acid products synthesized by de novo FAS (i.e., fatty acids of $\leq 18$ carbon atoms) (Fig. 4a and b).

342 However, when one considers the products of the FAE system, there are considerable

343 quantitative and qualitative difference in the VLCFAs produced by these ZmKCS-

344 complementing strains (Fig. 3c and d). Specifically, complementation by ZmKCS1, ZmKCS15,

345 and $\mathrm{ZmKCS} 20$ results in a $\sim 2$ - to 4-fold increase in the accumulation of VLCFAs as compared to

346 the control strains (Fig. 3c). In contrast, whereas both ZmKCS2 and ZmKCS4 complement the

347 lethality of the scelo2; scelo3 double mutant, the resulting strains generate quantities of VLCFAs

348 that resemble the levels seen in the three control strains. Moreover, each of these recovered

349 strains generated VLCFA profiles that are distinct from each other, and markedly different from

350 the profiles of the three control strains (Fig. 4d). Principal component analysis (PCA) of these

351 data identifies two principal components that together account for $\sim 70 \%$ of the variation in the

352 data (Fig. 5). These analyses indicate that whereas the variance in the VLCFAs of the pScELO3-

353 expressing recipient strain correlates to the BY4741 and BY4742 WT strains, all five ZmKCS

354 complemented strains present distinct FAE product profiles. Furthermore, these analyses

355 separate the strains based upon their VLCFA profiles (PCA1) or based upon the profiles of the

356 hydroxylated VLCFAs (PCA2). Specifically, the ZmKCS11 and ZmKCS20 complementing

357 strains vary from the control strains primarily in the VLCFA profiles, whereas the ZmKCS2,

$358 \mathrm{ZmKCS4}$ and $\mathrm{ZmKCS15}$ complementing strains vary from the control strains primarily in the 359 profiles of the hydroxylated VLCFAs.

361 More specific examination of the FAE generated products identify that whereas the control

362 strains produce predominantly 26-carbon VLCFAs and the 2-hydroxy derivative, only the

$363 Z m K C S 15$-complementing strain generates these VLCFAs. The ZmKCS2 complement 
364 predominantly produces 20 -carbon fatty acid and cannot elongate fatty acids beyond 22-carbon

365 chain length. The ZmKCS4 complement accumulates products with chain lengths ranging from

36620 to 24-carbons but is unable to elongate beyond this chain length. Both the ZmKCS11- and

367 ZmKCS20-complementing strains produce abundant quantities of both 20- and 22-carbon

368 products and can only generate smaller quantities of 24-carbon VLCFAs.

\section{Discussion}

372 Although VLCFAs account for a relatively small portion $(<10 \%)$ of the fatty acids that constitute

373 cellular lipids, their significance to biological processes is illustrated by their essentiality to

374 viability. Namely, mutations that block the ability to elongate preexisting fatty acids to VLCFAs

375 present a lethal phenotype in a wide range of phylogenetic clades of eukaryotes ${ }^{8,9,16,30,31}$. The

376 length of these molecules provides chemophysical characteristics that make them important

377 components of phospholipids, sphingolipids and glycerolipids within biological membranes.

378 Moreover, in plants these molecules also have a unique role in being precursors to the cuticle, the

379 coating that covers the aerial surfaces of these organisms ${ }^{3,4,6}$. Evolutionarily, the assembly of

380 the cuticle appeared about $450 \mathrm{MYA}$ and allowed plants to adapt to life on land, a desiccating

381 environment, as compared to the aquatic environment where life is thought to have first arisen ${ }^{6}$.

383 The isolation and characterization of the enzymatic components of plant FAE system ${ }^{8,13,18}$ has

384 identified a dilemma concerning the significance of the biochemical and genetic redundancy in

385 the enzymes that catalyze some of the reactions of the FAE system. In particular, two distinct 386 enzymes catalyze the Claisen condensation reaction of the FAE cycle, the KCS-type enzymes

387 that occur uniquely in plants, and the ELO-type enzymes that are phylogenetically more widely 
388 distributed ${ }^{18}$. Moreover, plant genomes contain a large number of ELO genes and even larger

389 number of $K C S$ genes ${ }^{18,32-35}$. Although this appears to be a characteristic widely dispersed

390 among plant phyla, it is not as prevalent among the other components of the FAE system. Thus,

391 although single copy genes encode the HCD and ECR components in maize and Arabidopsis

$39218,36,37$, the maize genome carries two paralogs of the KCR component ${ }^{8}$, whereas the Arabidopsis

393 genome encodes only one functional copy of this enzyme 9 .

395 Addressing this redundancy dilemma, either by in vivo or in vitro strategies, presents several 396 challenges, primarily associated with the integral membrane nature of the FAE system, which 397 makes it difficult to isolate these proteins in their native conformation. Additionally, the number 398 of gene paralogs and the functional redundancy within the FAE system complicate the use of 399 forward or reverse genetics strategies to decipher function. In this study we have used an

400 alternative approach, employing heterologous expression in the yeast $S$. cerevisiae, to 401 characterize the $26 \mathrm{ZmKCS}$ paralogs that are identifiable in the maize genome.

\section{Insights on the molecular organization of the FAE system}

404 Initial characterizations involved the individual expression of the $26 \mathrm{ZmKCS}$ paralogs in a yeast 405 strain that carried the endogenous wild-type FAE system; this genetic modification had minor 406 changes on the attributes that we quantified (i.e., cell growth or fatty acid profiles). Subsequent 407 experiments involved the expression of these ZmKCS paralogs in recipient mutant strain that did 408 not express the FAE components that catalyze the Claisen-condensation reaction of the yeast 409 system. Yeast carries three genes that encode this catalytic function (ScELO1, ScELO2 and 410 ScELO3), and we focused on the latter 2 paralogs because $S c E L O 1$ elongates preexisting fatty 
411 acids to only 18 -carbon chain length ${ }^{7,17}$. However, both ScELO2 and ScELO3 can produce

412 VLCFAs, with the former being capable of producing 24-carbon fatty acids, and the latter is

413 required for the synthesis of 26-carbon fatty acids ${ }^{7,38,39}$. Moreover, whereas each scelo2 and

414 scelo3 single mutants are viable, the scelo2; scelo3 double mutant exhibits synthetic lethality ${ }^{16}$.

416 The individual scelo2 and scelo3 mutations had opposite quantitative effects in reducing and 417 increasing the accumulation of VLCFAs, respectively. Additionally, only the scelo3 mutant 418 changed the chain-length profile of the VLCFA products. Yet, the expression of each of the 419 ZmKCS proteins in these mutant strains did not substantially alter the fatty acid profiles from 420 those expressed by each single mutant strain. Collectively therefore, these three sets of 421 expression experiments suggest that the ZmKCS components are unable to displace the $S c$ ELO 422 components from the $S c$ FAE complex, even when the $S c$ FAE system is disrupted by the absence 423 of one of the three $S c$ ELO proteins that catalyzes the Claisen-condensation reaction required by 424 the native yeast FAE system. This conclusion is consistent with prior studies ${ }^{39,40}$, which found 425 that the yeast FAE system can be co-immunoprecipitated as a partial complex. Moreover, one 426 could speculate that our findings are indicative of a FAE quaternary model in which there are 427 distinct $S c E L O 2-$ and $S c E L O 3$-containing FAE complexes, and thus the individual scelo2 or 428 scelo3 mutations would not disrupt a mixed $S c$ ELO2/ScELO3-containing FAE complex, but 429 individually eliminates one of these distinct complexes. Consistent with this supposition, global 430 interactome data of integral membrane proteins in yeast did not provide evidence for ScELO2 431 and ScELO3 interactions ${ }^{41}$, though these interactome data identify interactions of these two 432 proteins with other $S c$ FAE components ${ }^{39,40}$. 
434 This supposition was further explored in a third series of experiments in which we expressed 435 each ZmKCS in a scelo2; scelo3 double mutant strain. Such a strain is synthetically lethal, but

436 its viability was maintained by an ectopic copy of a wild-type ScELO3 allele. A plasmid-shuffle

437 strategy was used to identify five ZmKCS paralogs that are capable of replacing the ectopic

438 ScELO3 allele and maintaining the viability of the scelo; scelo3 double mutant. The specific

439 complementing isozymes being ZmKCS2, ZmKCS4, ZmKCS11, ZmKCS15 and ZmKCS20.

440 These five ZmKCS isozymes that can complement the lethality of the scelo2; scelo3 double

441 mutant belong to two different previously defined phylogenetic KCS-clades, the $\alpha / \beta$ and the $\zeta$

442 clades ${ }^{18,33}$. Moreover, these $Z m$ KCS proteins are inclusive of all members of the $\zeta$ clade (i.e.,

$443 Z m K C S 2, Z m K C S 11$, and ZmKCS20) and all members of the $\alpha / \beta$ clade (ZmKCS4 and

$444 \mathrm{ZmKCS15).} \mathrm{Because} \mathrm{primary} \mathrm{structure} \mathrm{homology} \mathrm{defines} \mathrm{these} \mathrm{clades,} \mathrm{one} \mathrm{could} \mathrm{surmise}$

445 therefore that the $\mathrm{ZmKCS}$ proteins in these two clades share common structural feature(s) that

446 facilitates interactions with the other components of the yeast FAE system, and that these

447 structural feature(s) are absent from the other $21 \mathrm{ZmKCSs}$ that belong to the other 7 distinct

448 phylogenetic clades, which cannot achieve genetic complementation.

\section{KCS components as determinants of FAE product profiles}

451 It's generally believed that the product profile of the FAE system is a property of the enzyme 452 component that catalyzes the Claisen-condensation reaction of each FAE cycle. Although

453 evidence for this supposition has been gathered for the ELO proteins $16,17,39,42,43$ and KCS

454 proteins ${ }^{21,34,44-50}$, this study, in combination with the prior characterizations of $Z m \operatorname{KCS} 4{ }^{18}$ and

$455 \mathrm{ZmKCS} 19^{51}$, provides a systematic assessment of this hypothesis for the KCS-class of FAE

456 enzymes. The unique VLCFA profiles generated by the five complemented strains reveals 
457 differences in the enzymatic capability of each ZmKCS (Fig. 6). We find that the ZmKCS2 has

458 the ability to elongate fatty acids to 22-carbon, ZmKCS4, ZmKCS11, and ZmKCS20 has the

459 ability to elongate up to 24-carbons, and ZmKCS15 can elongate up to 26-carbons. One could

460 postulate that the production of 26-carbon products, which is the most abundant VLCFA in WT

461 yeast, contributes to the success of the complementation by ZmKCS15; this was the fastest

462 growing strain among the recovered complemented strains.

464 A significant proportion of the recovered VLCFAs are the 2-hydroxy-derivatives, which are 465 associated with the ceramide moiety of sphingolipids. In yeast cells sphingolipids make up 10$46620 \%$ of the membrane lipids ${ }^{16}$, and these complex lipids serve vital requirements for cellular 467 viability ${ }^{52}$. As with prior studies ${ }^{28}$, we found that in WT yeast the dominant hydroxy-fatty acid 468 incorporated into the ceramide moiety of the sphingolipids is 2-hydroxy-26:0, accounting for 469 over $70 \%$ of the fatty acids that are associated with this lipid. The ZmKCS-complemented strains 470 express dramatic differences in the accumulation of 2-hydroxy-fatty acid products. Although,

471 the near absence of 2-hydroxy-VLCFAs does not affect the viability of these cells, they do suffer

472 a severe growth penalty. Thus, these strains provide a synthetic biology platform to further 473 explore the dependency between VLCFAs and 2-hydroxy-fatty acid products and the physiology 474 of this organism.

476 Additionally, the strains we generated in this study are beyond the capabilities of natural 477 evolutionary processes and can provide deeper insights on the substrate specificity and VLCFA478 products generation by ZmKCS-containing FAE systems. For example, in planta the maize FAE 479 system has the ability to generate VLCFAs of up to 32 and 34 carbons ${ }^{8,51}$. Yet even though KCS 
appears to be the determinant of the chain length that FAE can generate, the systems we have

481 assembled with ZmKCS enzymes do not recapitulate this in planta metabolic capability. There

482 are one or more explanations for this apparent deficiency of the yeast system, including the

483 possibility that this is an attribute associated with the other ZmKCS paralogs that did not

484 complement the scelo2; scelo3 double mutant lethality, or the involvement of ZmELO proteins.

485 Additionally, analysis of plant mutants indicate that the ZmKCR components can also affect the

486 FAE product profile ${ }^{8}$. Furthermore, in planta evidence indicates that genetically encoded

487 cofactors (e.g., Glossy2 and Glossy2-like) may enhance the catalytic capability of the terminal

488 elongation cycles of FAE ${ }^{53}$. These hypotheses can now be directly evaluated by using the yeast

489 platform that we have developed in this study. Specifically, the ZmKCS-complementing strains

490 represent a new genetic resource to further explore these questions by individually co-expressing

$491 Z m K C R$ isozymes or the ZmGL2 coenzymes and directly evaluating how these additional

492 components provide the plant FAE system with the dexterity to generate diverse VLCFA

493 products needed for many metabolic endpoints that include the cuticle, seed oils, and membrane

494 lipids (sphingolipids and phospholipids).

495

496 References

497 1. Leonard, A. E., Pereira, S. L., Sprecher, H. \& Huang, Y.-S. Elongation of long-chain fatty

498 acids. Prog. Lipid Res. 43, 36-54 (2004).

499 2. Ǩezanka, T. Very-long-chain fatty acids from the animal and plant kingdoms. Prog. Lipid

$500 \quad$ Res. 28, 147-187 (1989).

501 3. Bach, L. \& Faure, J.-D. Role of very-long-chain fatty acids in plant development, when

502 chain length does matter. C. R. Biol. 333, 361-70 (2010). 
503 4. Haslam, T. M. \& Kunst, L. Extending The Story Of Very-Long-Chain Fatty Acid

504 Elongation. Plant Science. 210, 93-107 (2013).

505 5. Jenks, M. A. et al. Chemically Induced Cuticle Mutation Affecting Epidermal Conductance 506 to Water Vapor and Disease Susceptibility in Sorghum bicolor (L.) Moench. Plant Physiol. $507 \quad \mathbf{1 0 5}, 1239-1245(1994)$.

508 6. Yeats, T. H. \& Rose, J. K. C. The Formation and Function of Plant Cuticles. Plant Physiol. $509 \quad \mathbf{1 6 3}, 5-20(2013)$.

510 7. Oh, C.-S. S., Toke, D. A., Mandala, S. \& Martin, C. E. ELO2 and ELO3, Homologues of 511 theSaccharomyces cerevisiae ELO1 Gene, Function in Fatty Acid Elongation and Are 512 Required for Sphingolipid Formation. J. Biol. Chem. 272, 17376-17384 (1997).

513 8. Dietrich, C. R. et al. Characterization of two GL8 paralogs reveals that the 3-ketoacyl 514 reductase component of fatty acid elongase is essential for maize (Zea mays L.)

515 development. Plant J. 42, 844-861 (2005).

516 9. Frédéric Beaudoin. et al. Functional characterization of the Arabidopsis beta-ketoacyl517 coenzyme A reductase candidates of the fatty acid elongase. Plant Physiol. 150, 1174-91 518 (2009).

519 10. Harwood, J. L. Fatty acid metabolism. Ann Rev Plant Physiol 39, 101-138 (1988).

520 11. Harwood, J. L. Recent advances in the biosynthesis of plant fatty acids. Biochim. Biophys. $521 \quad$ Acta - Lipids Lipid Metab. 1301, 7-56 (1996).

522 12. Ohlrogge, J. B. \& Jaworski, J. G. REGULATION OF FATTY ACID SYNTHESIS. Annu. 523 Rev. Plant Physiol. Plant Mol. Biol. 48, 109-136 (1997).

524 13. Kunst, L., Taylor, D. C. \& Underhill, E. W. Fatty acid elongation in developing seeds of 525 Arabidopsis thaliana. Plant Physiol Biochem 30, 425-434 (1992). 
526 14. Lemieux, B., Miquel, M., Somerville, C. \& Browse, J. Mutants of Arabidopsis with

527 alterations in seed lipid fatty acid composition. Theor. Appl. Genet. 80, 234-240 (1990).

528 15. James, D. W. \& Dooner, H. K. Isolation of EMS-induced mutants in Arabidopsis altered in

529 seed fatty acid composition. Theor. Appl. Genet. 80, 241-245 (1990).

530 16. Oh, C.-S., Toke, D. A., Mandala, S. \& Martin, C. E. ELO2 and ELO3, Homologues of the

531 Saccharomyces cerevisiae ELO1 Gene, Function in Fatty Acid Elongation and Are Required 532 for Sphingolipid Formation. J. Biol. Chem. 272, 17376-17384 (1997).

533 17. Toke, D. A. \& Martin, C. E. Isolation and Characterization of a Gene Affecting Fatty Acid

534 Elongation in Saccharomyces cerevisiae. J. Biol. Chem. 271, 18413-22 (1996).

535 18. Campbell, A. A. et al. A single-cell platform for reconstituting and characterizing fatty acid 536 elongase component enzymes. PLOS ONE. 14, e0213620 (2019).

537 19. Jakobsson, A., Westerberg, R. \& Jacobsson, A. Fatty acid elongases in mammals: Their 538 regulation and roles in metabolism. Prog. Lipid Res. 45, 237-249 (2006).

20. Guo, H.-S. et al. Evolution of the KCS gene family in plants: the history of gene duplication, sub/neofunctionalization and redundancy. Mol. Genet. Genomics. 291, 739-52 (2016).

21. Xiao, G.-H., Wang, K., Huang, G. \& Zhu, Y.-X. Genome-scale analysis of the cotton KCS

22. Altschul, S. F., Gish, W., Miller, W., Myers, E. W. \& Lipman, D. J. Basic local alignment gene family revealed a binary mode of action for gibberellin A regulated fiber growth. $J$. search tool. J. Mol. Biol. 215, 403-10 (1990).

23. Alberti, S., Gitler, A. D. \& Lindquist, S. A suite of Gateway® cloning vectors for highthroughput genetic analysis in Saccharomyces cerevisiae. Yeast 24, 913-919 (2007).

548 24. Sherman, F., Fink, G. \& Hicks, J. Methods in Yeast Genetics. (1986). 
25. Gietz, R. D. \& Woods, R. A. Transformation of yeast by lithium acetate/single-stranded carrier DNA/polyethylene glycol method. Methods Enzymol. 350, 87-96 (2002).

551 26. Enyenihi, A. H. \& Saunders, W. S. Large-scale functional genomic analysis of sporulation 552 and meiosis in Saccharomyces cerevisiae. Genetics. 163, 47-54 (2003).

553 27. Truong, D. M. \& Boeke, J. D. Resetting the Yeast Epigenome with Human Nucleosomes. $554 \quad$ Cell. 171, 1508-1519.e13 (2017).

555 28. Ejsing, C. S. et al. Global analysis of the yeast lipidome by quantitative shotgun mass 556 spectrometry. Proc. Natl. Acad. Sci. 106, 2136-2141 (2009).

557 29. Tehlivets, O., Scheuringer, K. \& Kohlwein, S. D. Fatty acid synthesis and elongation in yeast. Biochimica et Biophysica Acta - Molecular and Cell Biology of Lipids. 1771, 255-270

30. Sassa, T. et al. Impaired Epidermal Permeability Barrier in Mice Lacking Elovl1, the Gene Responsible for Very-Long-Chain Fatty Acid Production. Mol. Cell. Biol. 33, 2787 LP 2796 (2013).

31. Vasireddy, V. et al. Loss of functional ELOVL4 depletes very long-chain fatty acids (> or =C28) and the unique omega-O-acylceramides in skin leading to neonatal death. Hum. Mol. Genet. 16, 471-82 (2007).

32. Guo, H. S. et al. Evolution of the KCS gene family in plants: the history of gene duplication,

568 33. Joubès, J. et al. The VLCFA elongase gene family in Arabidopsis thaliana: phylogenetic 569 analysis, 3D modelling and expression profiling. Plant Mol. Biol. 67:547-566 (2008) . 
34. Huai, D. et al. Genome-Wide Identification of Peanut KCS Genes Reveals That AhKCS1 and AhKCS28 Are Involved in Regulating VLCFA Contents in Seeds. Front. Plant Sci. 11, $1-18(2020)$.

35. Wang, Q. et al. Functional identification of ELO-like genes involved in very long chain fatty acid synthesis in Arabidopsis thaliana. Russ. J. Plant Physiol. 61, 853-861 (2014).

36. Bach, L. et al. The very-long-chain hydroxy fatty acyl-CoA dehydratase PASTICCINO2 is essential and limiting for plant development. Proc. Natl. Acad. Sci. 105, 14727-31 (2008).

37. Zheng, H., Rowland, O. \& Kunst, L. Disruptions of the Arabidopsis Enoyl-CoA reductase gene reveal an essential role for very-long-chain fatty acid synthesis in cell expansion during plant morphogenesis. Plant Cell. 17, 1467-81 (2005).

38. Rössler, H., Rieck, C., Delong, T., Hoja, U. \& Schweizer, E. Functional differentiation and selective inactivation of multiple Saccharomyces cerevisiae genes involved in very-longchain fatty acid synthesis. Mol. Genet. Genomics. 269, 290-8 (2003).

39. Denic, V. \& Weissman, J. S. A molecular caliper mechanism for determining very longchain fatty acid length. Cell. 130, 663-77 (2007).

40. Han, G. et al. The Saccharomyces cerevisiae YBR159w gene encodes the 3-ketoreductase of the microsomal fatty acid elongase. J. Biol. Chem. 277, 35440-9 (2002).

41. Miller, J. P. et al. Large-scale identification of yeast integral membrane protein interactions. Proc. Natl. Acad. Sci. 102, 12123-12128 (2005).

42. Jump, D. B. Mammalian fatty acid elongases. Methods Mol. Biol. 579, 375-389 (2009).

591 43. Matsuzaka, T. et al. Hepatocyte ELOVL Fatty Acid Elongase 6 Determines Ceramide Acyl592 Chain Length and Hepatic Insulin Sensitivity in Mice. Hepatology. 71, 1609-1625 (2020). 
44. Paul, S., et al. Members of the Arabidopsis FAE1-like 3-Ketoacyl- CoA Synthase Gene

594 Family Substitute for the Elop Proteins of Saccharomyces cerevisiae. J. Biol. Chem. 281, 595 9018-29 (2006).

596 45. Blacklock, B. J. \& Jaworski, J. G. Substrate specificity of Arabidopsis 3-ketoacyl-CoA 597 synthases. Biochem. Biophys. Res. Commun. 346, 583-590 (2006).

598 46. Trenkamp, S., Martin, W. \& Tietjen, K. Specific and differential inhibition of very-long599 chain fatty acid elongases from Arabidopsis thaliana by different herbicides. Proc. Natl. $600 \quad$ Acad. Sci. 101, 11903-8 (2004).

601 47. Daniela, H. et al. Arabidopsis ketoacyl-CoA synthase 16 (KCS16) forms C36/C38 acyl

602 precursors for leaf trichome and pavement surface wax. Plant Cell Environ. 40, 1761-1776 $603 \quad$ (2017).

604 48. Kim, J. et al. Arabidopsis 3-ketoacyl-coenzyme a synthase9 is involved in the synthesis of 605 tetracosanoic acids as precursors of cuticular waxes, suberins, sphingolipids, and 606 phospholipids. Plant Physiol. 162, 567-80 (2013).

607 49. Kim, J., Lee, S. B. \& Suh, M. C. Arabidopsis 3-Ketoacyl-CoA Synthase 4 is Essential for 608 Root and Pollen Tube Growth. J. Plant Biol. 64, 155-165 (2021).

609 50. Lassner, M. W., Lardizabal, K. \& Metz, J. G. A jojoba beta-Ketoacyl-CoA synthase cDNA 610 complements the canola fatty acid elongation mutation in transgenic plants. Plant Cell. 8, $611 \quad 281-92(1996)$.

612 51. Liu, X. et al. The FUSED LEAVES1-ADHERENT1 Regulatory Module Is Required For 613 Maize Cuticle Development And Organ Separation. New Phytologist. 229, 388-402 (2021).

614 52. Cerantola, V. et al. Yeast sphingolipids do not need to contain very long chain fatty acids. 615 Biochem. J. 401, 205-216 (2006). 
616 53. Alexander, L. E. et al. Maize Glossy2 and Glossy2-like Genes Have Overlapping and

617 Distinct Functions in Cuticular Lipid Deposition. Plant Physiol. 183, 840-853 (2020).

618

619 Data Availability

620 Stenback, KE., Flyckt, KS., Hoang, T., Campbell AC., \& Nikolau, BJ.. Fatty acid profiles of

621 yeast strains individually expressing 26 Zea mays KCS isozymes. Iowa State University public

622 data repository: $10.25380 /$ iastate. 17131460

623

624 Stenback, KE., Flyckt, KS., Hoang, T., Campbell AC., \& Nikolau, BJ.. Growth data of yeast

625 strains individually expressing 26 Zea mays KCS isozyme. Iowa State University public data

626 repository: 10.25380/iastate. 17131460

627

628 Stenback, KE., Flyckt, KS., Hoang, T., Campbell AC., \& Nikolau, BJ.. Cell wall thickness of

629 yeast mutant strains genetically complemented by the expression of Zea mays KCS isozymes.

630 Iowa State University public data repository: 10.25380/iastate.17131460

631

632 For review, please access the data using the following link:

633 https://figshare.com/s/298a83d8d29b063dd299

634

\section{Acknowledgements}

636 The authors acknowledge Dr. Ann Perera and Dr. Lucas Showman of the WM Keck

637 Metabolomics Research Laboratory (Iowa State University) for assistance in conducting the

638 metabolic profiling studies. They also acknowledge Tracey Pepper Stewart of the High 
639 Resolution Microscopy Facility (Iowa State University) for assistance with the transmission

640 electron microscopy studies. This study was partially supported by grants from the National

641 Science Foundation (IOS 1354799 and EEC 0813570), Hatch Project No. IOW03802 from the

642 United Sates Department of Agriculture's National Institute of Food and Agriculture, and Iowa

643 State University's Center for Metabolic Biology.

\section{Author Contributions}

645 B.J.N and A.A.C conceived the study. K.E.S, K.S.F, and T.H contributed to strain generation.

646 K.E.S and K.S.F characterized the yeast strains by acquiring and analyzing fatty acid

647 composition and growth-rate data. K.E.S acquired and analyzed the morphological data. B.J.N

648 and K.E.S. led manuscript writing with contributions from all authors.

\section{Competing Interests}

650 The authors declare no competing interests. 


\section{Figures}

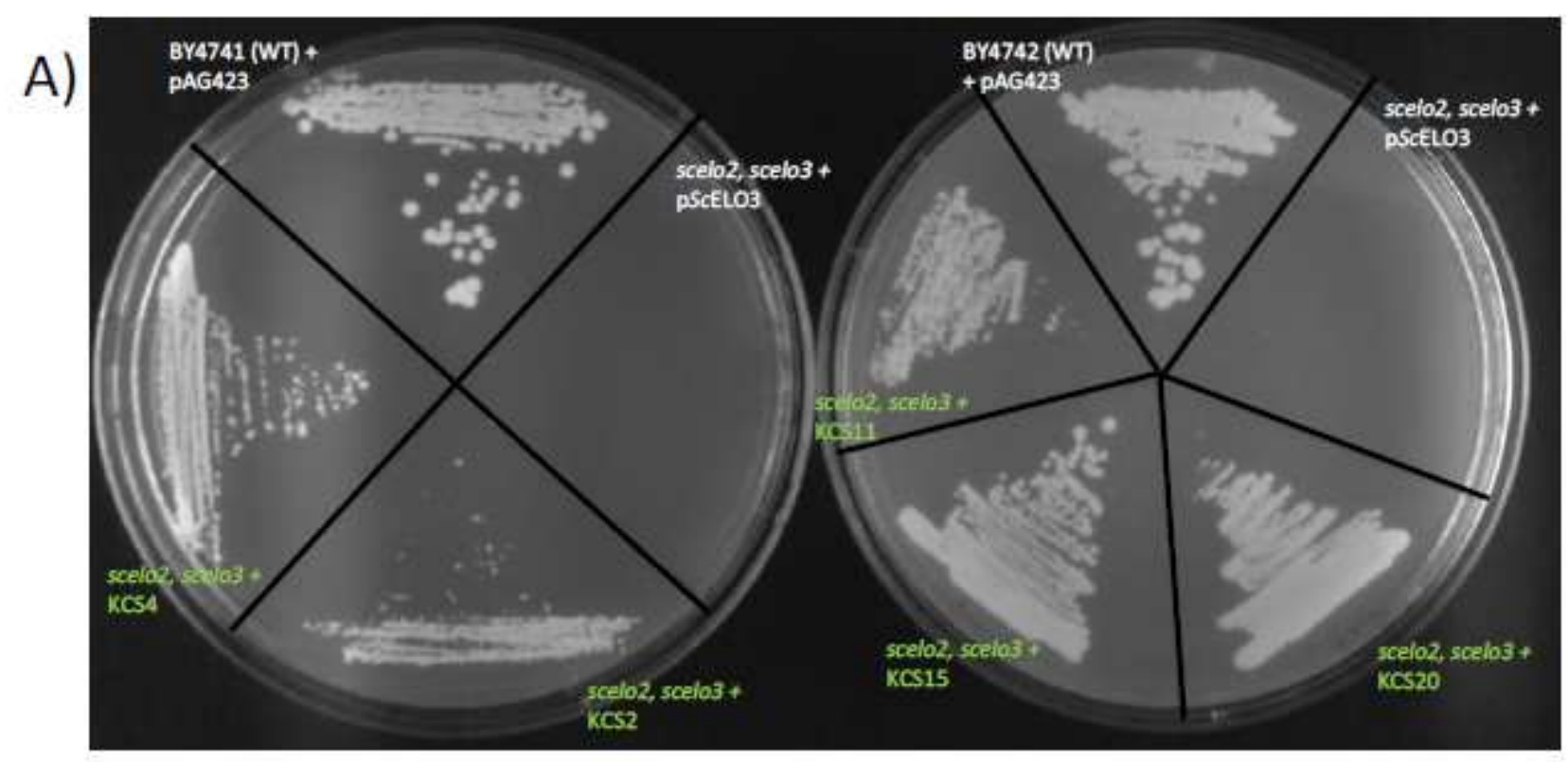

B)

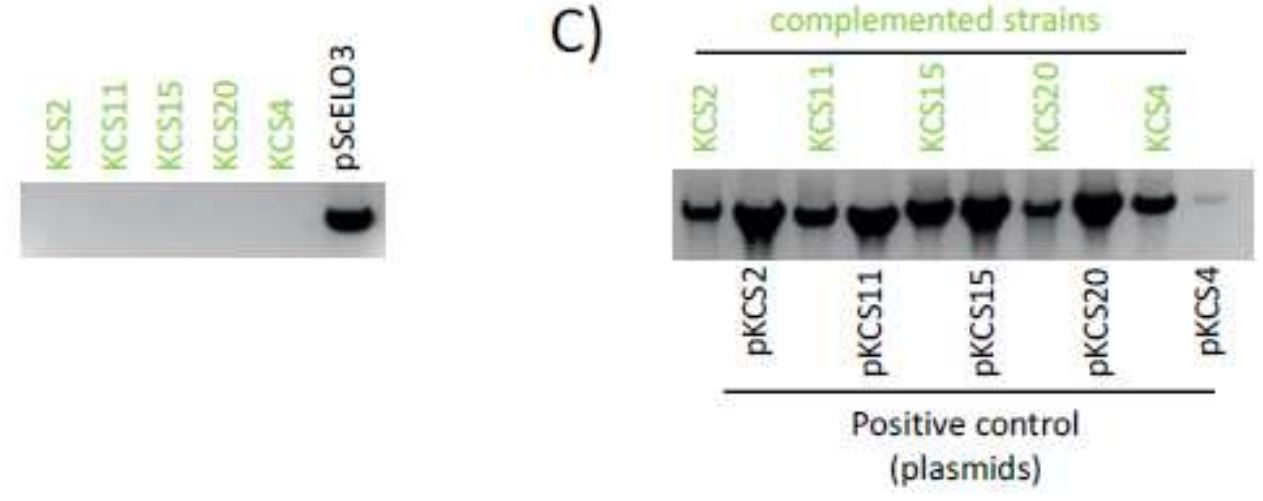

\section{Figure 1}

ZmKCS-expressing strains complement the lethality of the scelo2; scelo3 double mutant. Out of the 26 ZmKCSs evaluated, the expression of five ZmKCS isozymes were able to restore the viability of the synthetically lethal phenotype of the scelo2; scelo3 double mutant strain. A) Growth of the indicated strains on SD his- media containing 5-FOA; controls include the WT BY4741 and BY4742 strains harboring the empty PAG423-GPD vector, and the scelo2; scelo3 double mutant strain whose viability was maintained by the ectopic expression of ScELO3. B) PCR molecular confirmation of the absence of the ectopic ScELO3 in the recovered ZmKCS complemented strains. C) PCR molecular confirmation of the presence of the $\mathrm{ZmKCS}$ sequence in the recovered $\mathrm{ZmKCS}$ complemented strains. 


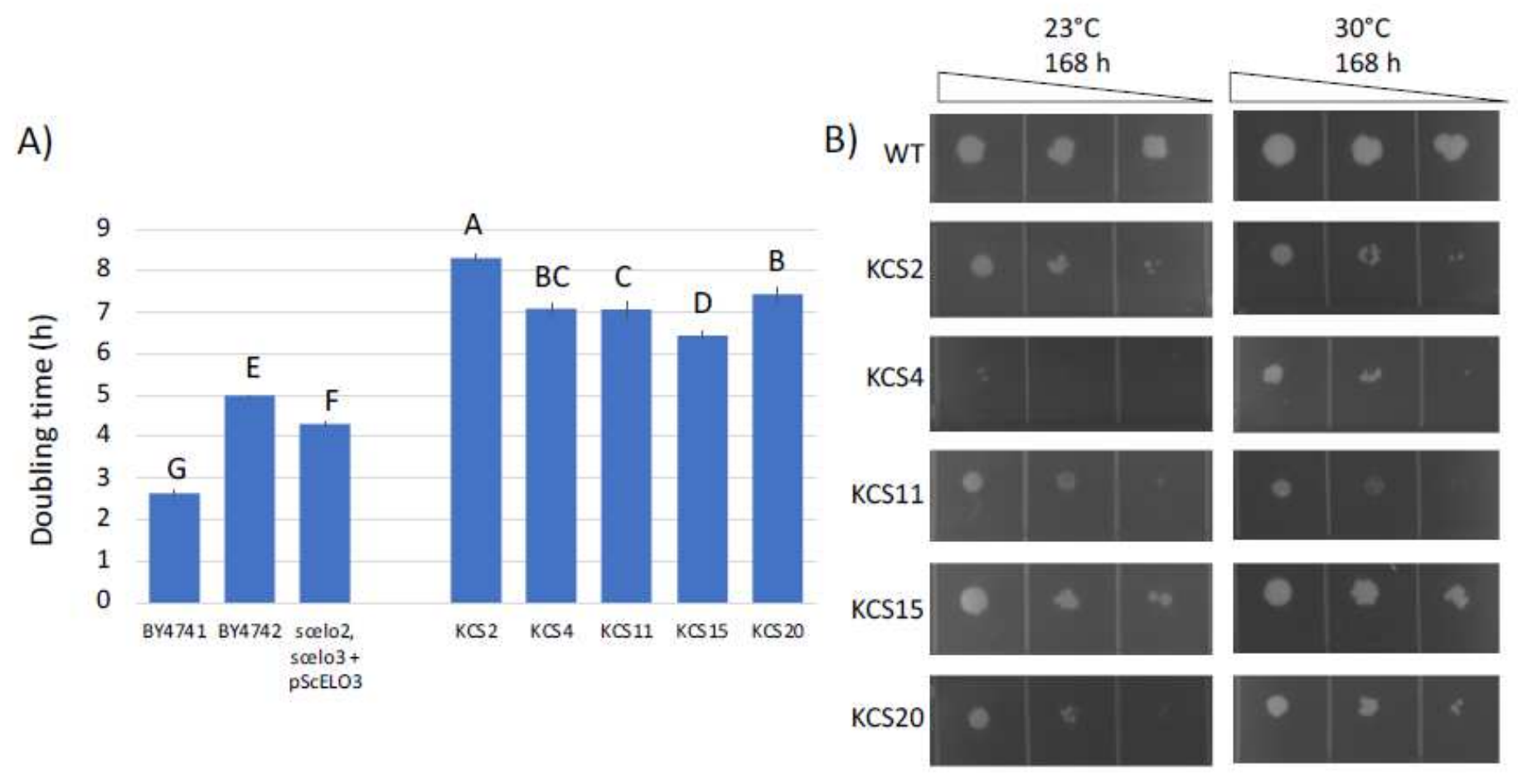

Figure 2

Growth of the ZmKCS complementing strains. A) Culture doubling time of the scelo2; scelo3 double mutant strain complemented by the expression of ZmKCS isozymes. Error bars represent standard error from 3 replicates, different letters above the data-bars indicate statically significant differences based on Student's t-test $(p<0.05)$. B) Serial 10 -fold dilution inoculum of each indicated strain was applied on solid media, and growth was evaluated at $23^{\circ} \mathrm{C}$ and $30^{\circ} \mathrm{C}$ at the indicated times after inoculation. Images are representative from three repetitions of this experiment. 

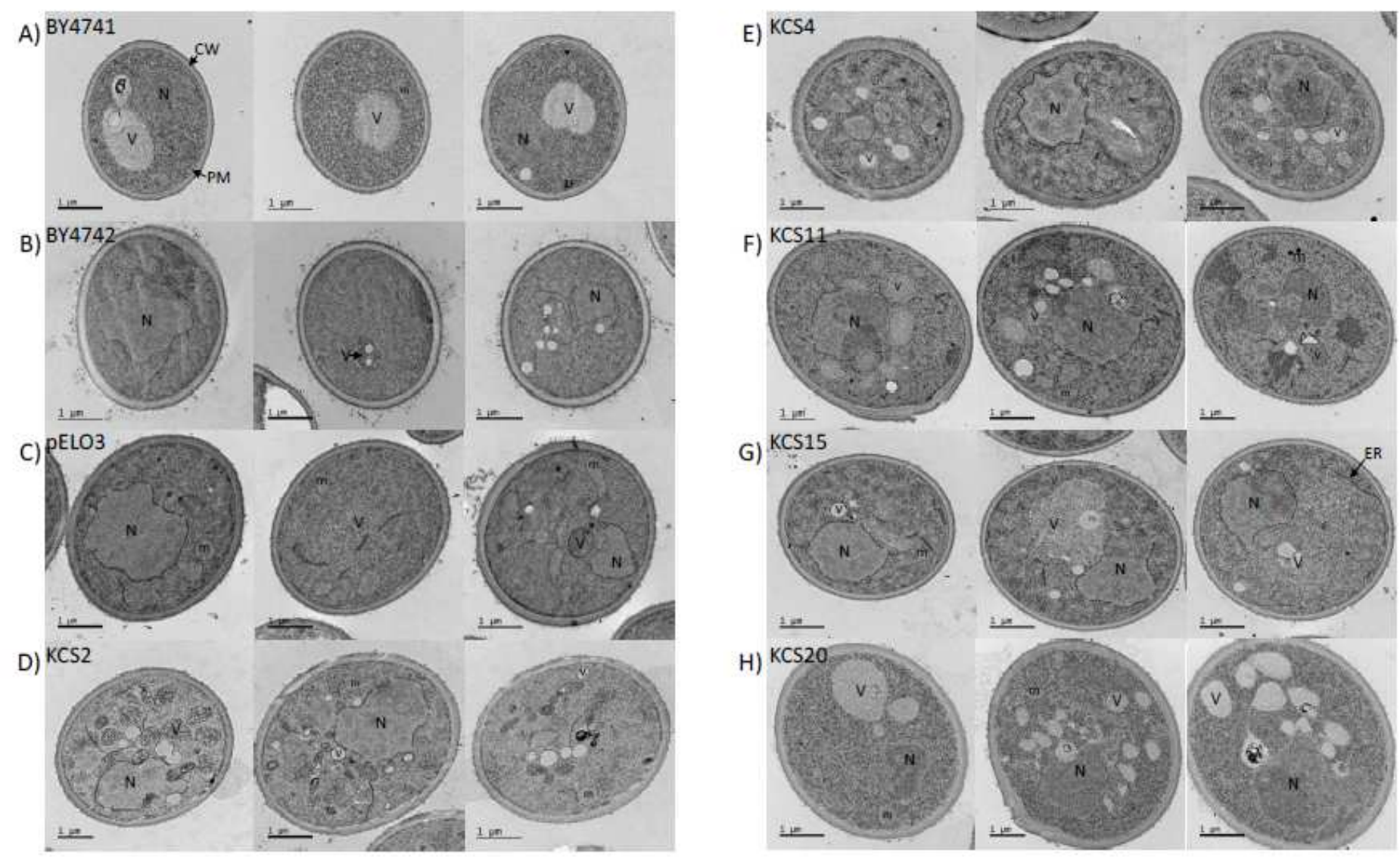

\section{Figure 3}

Ultrastructural comparison of ZmKCS complementing strains. Transition electron micrographs of three representative yeast cells of each identified genotype. $\mathrm{V}=$ vesicle, $\mathrm{m}=$ mitochondria, $\mathrm{N}=$ nucleus, $\mathrm{CW}=$ cell wall, $\mathrm{PM}=$ plasma membrane, $\mathrm{ER}=$ endoplasmic reticulum. Scale bars $=1 \mu \mathrm{m}$. 
A) B)
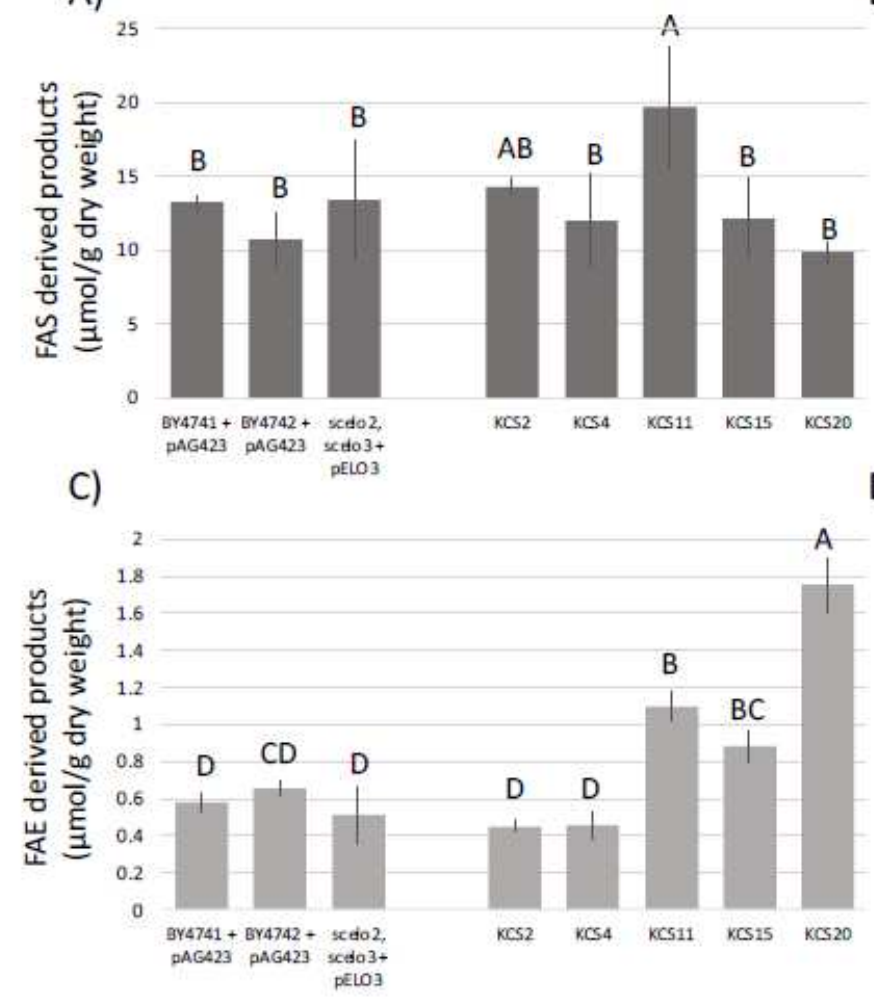

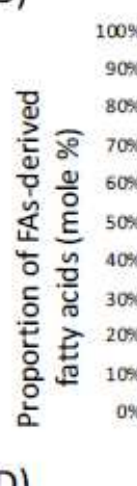

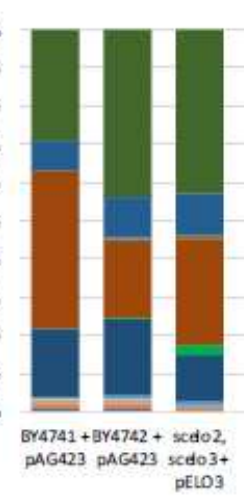

$100 \%$
돌:1

aC18:0-OH

mC18:0

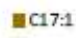

⿶C17:0

⿶C16:1

aC16:0-OH

- $16: 10$

- 15150

1) C14:1

inC14:0

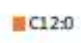

a $10: 5$

- $\mathrm{C} 26: 0-\mathrm{OH}$

든. 650

든 $\mathrm{C} 24 \mathrm{O}-\mathrm{OH}$

대 $24: 0$

둗.3.

들 $22.0-\mathrm{OH}$

$=022.0$

$=0210$

블.1

- 2020

\section{Figure 4}

Fatty acid profiles generated by the scelo2; scelo3 double mutant strains complemented by the expression of ZmKCS isozymes. A) Yield of FAS-derived products. B) Proportion of individual FAS-derived products. C) Yield of FAE-derived products. D) Proportion of FAE-derived products. See Methods for details on data gathering and analysis. Fatty acid profiles were determined by either GC-FID analysis of FAMEs. Error bars represent standard error from 3 replicates, and different letters above data-bars indicate statistically significant differences based on the Student's t-test $(p<0.05)$. 


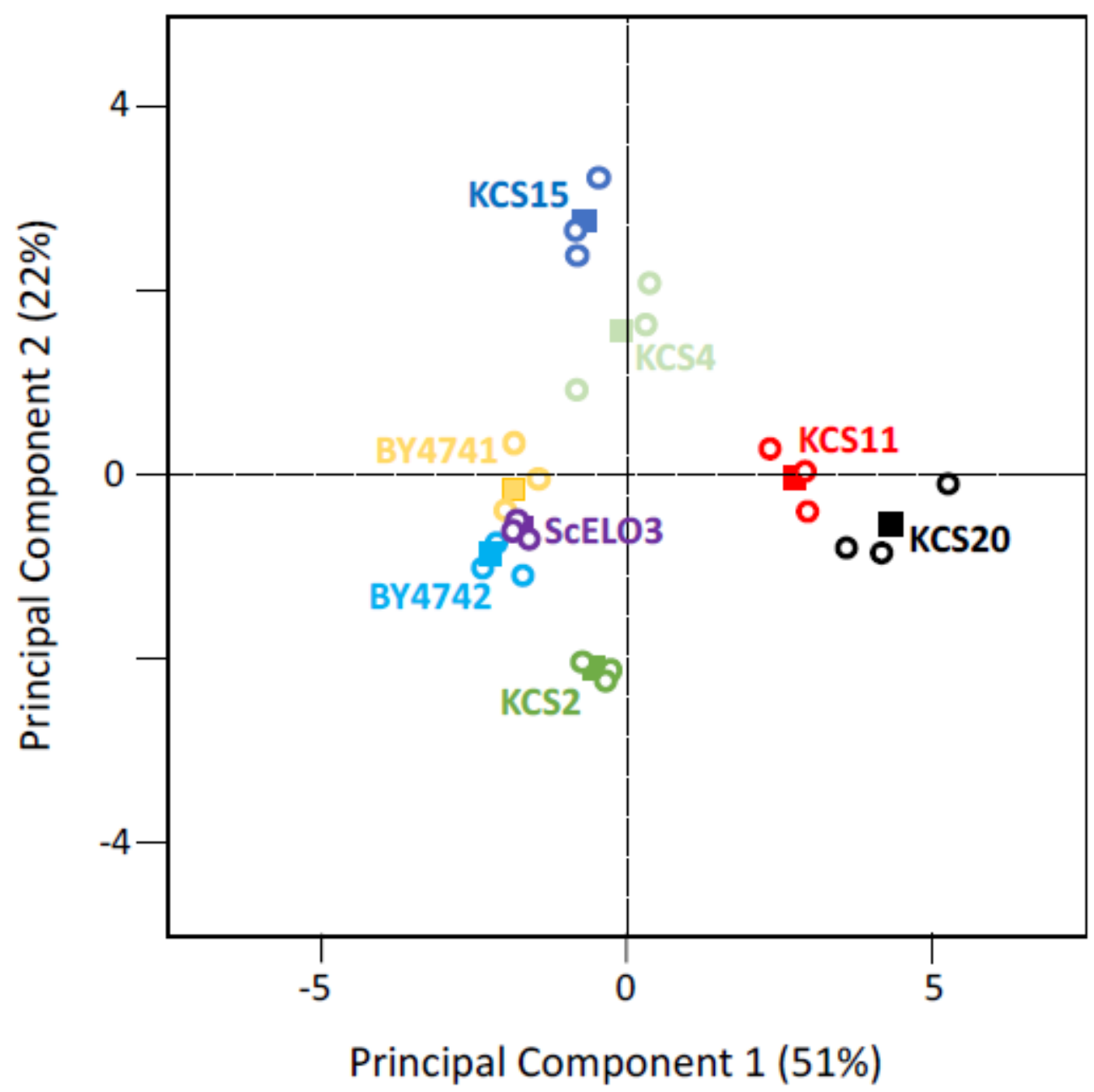

Figure 5

Principal component analysis (PCA) of VLCFA profiles produced by ZmKCS complementing strains. These analyses are of data presented in Figure 4C and D. Open circle data-points are from each of 3 replicate analyses of each genotype, and the average position of these data-points are displayed as the filled data-points. 


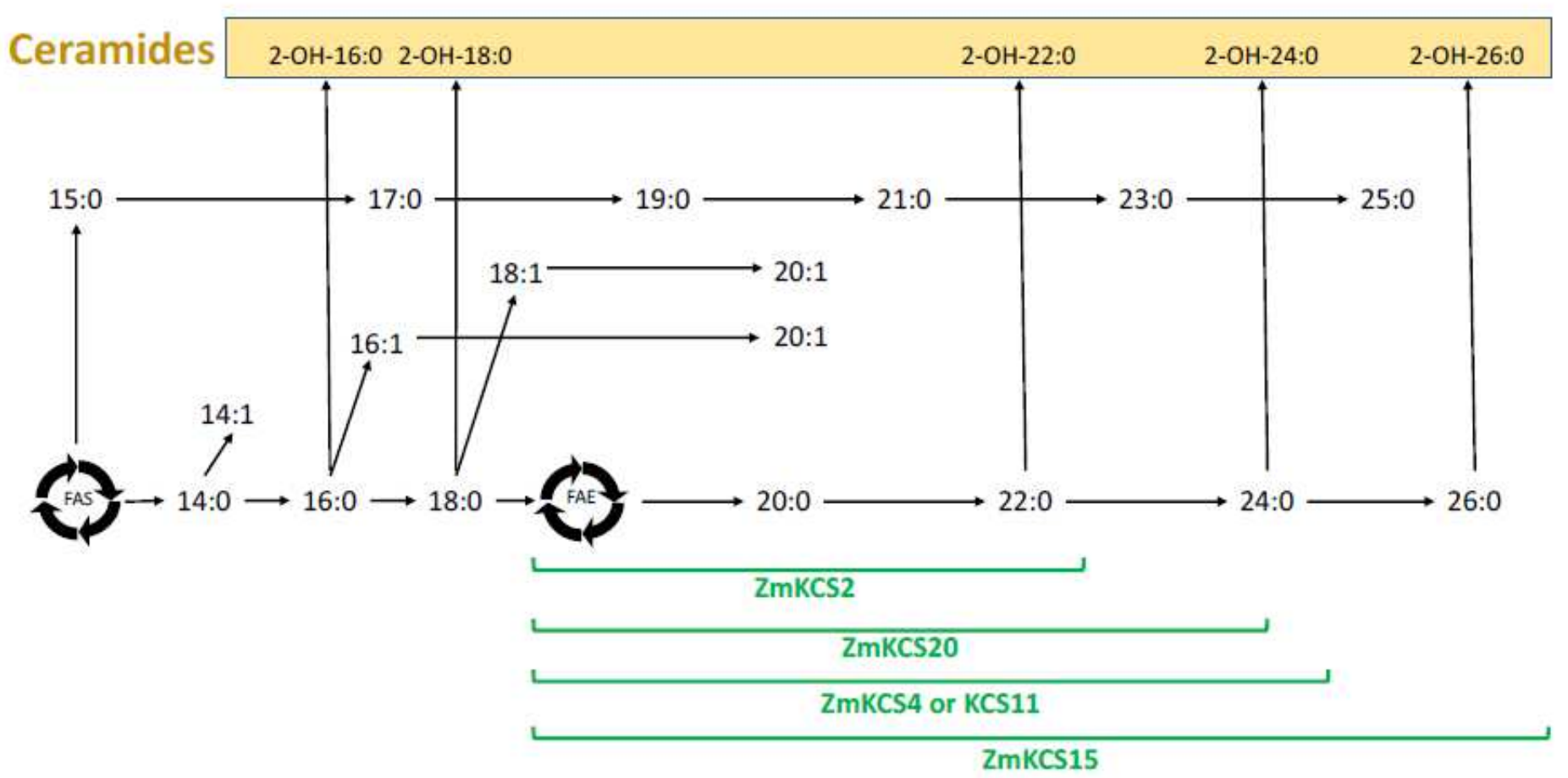

Figure 6

Enzymatic capabilities of the ZmKCS isozymes in generating VLCFAs. Metabolic model of the enzymatic capabilities of ZmKCS isozymes based on the VLCFA profiles expressed by the scelo2; scelo3 double mutant strains, complemented by the expression of different ZmKCS isozymes. FAS generated fatty acylCoAs of up to 18-carbon chain-length can be desaturated to generate monounsaturated fatty acids. FAE elongates these saturated and monounsaturated fatty acids to lengths of up to 26-carbons, and these fatty acids can be hydroxylated at the 2- position after they are incorporated into ceramide lipids. Green bars identify the differential enzymatic capability of each ZmKCS isozyme to catalyze elongation of fatty acids to specific chain lengths.

\section{Supplementary Files}

This is a list of supplementary files associated with this preprint. Click to download.

- Table1.pdf

- SupplementaryTable1.pdf

- SupplementaryTable2.pdf

- SupplementaryTable3.xlsx

- SupplementaryFig1.pdf

- SupplementaryFig2.pdf

- SupplementaryFig3.pdf

- SupplementaryFig4.pdf 
- SupplementaryFig5.pdf 